\title{
CONTRIBUIÇÕES DA FILOSOFIA PARA A PESQUISA EM ENFERMAGEM
}

\author{
Philosophy's contributions to research in Nursing \\ Contribuciones de la Filosofía para la investigación en Enfermería
}

Raquell Alves de Araújo ${ }^{1}$

Fátima Maria da Silva Abrão ${ }^{4}$
Hemília Gabrielly de Oliveira Cartaxo²

Antonio José de Almeida Filho 5
Samira Maria Oliveira Almeida ${ }^{3}$

Clara Maria Silvestre Monteiro de Freitas ${ }^{6}$

\section{RESUMO}

Objetivou-se realizar um levantamento das pesquisas, na área de Enfermagem, que fizeram uso da filosofia com perspectiva de compreender sua prática, buscando identificar sua contribuição para a profissão. Trata-se de um estudo cuja base metodológica é a revisão integrativa, de caráter crítico e retrospectivo, com fonte de dados secundários. Para a seleção dos artigos utilizaramse as bases de dados Lilacs e Medline. A amostra desta revisão constituiu-se de 31 artigos. Após análise dos artigos, os resultados apontaram que há uma dominação de estudos qualitativos e reflexivos, utilizando a fenomenologia como abordagem filosófica, predominando o referencial teórico do filósofo Martin Heidegger. Concluiu-se que a fenomenologia tem sido cada vez mais utilizada em trabalhos realizados no campo da Enfermagem, o que reflete a preocupação em compreender os fenômenos vividos no seu cotidiano. Assim, tem apresentado subsídios expressivos para a práxis da profissão e, especialmente, para a edificação do conhecimento enquanto disciplina.

Palavras-chave: Enfermagem. Filosofia. Pesquisa.

\begin{abstract}
The aim was to find the Nursing studies that used the Philosophy to understand its practice, and trying to identify its contributions to the profession. The methodology of this study is the integrative review, with critical and retrospective character. The databases used to select the articles were Lilacs and Medline. The sample was composed of 31 scientific articles. After analyzing them, the results showed that there were many qualitative and reflexive researches, using phenomenology as a philosophical approach, predominating the theoretical referential of the philosopher Martin Heidegger. We concluded that phenomenology has been used a lot in the Nursing articles. It reflects the intention to comprehend the phenomenon experienced in a daily life. Therefore, it has offered meaningful subsidies to the profession practice, and, especially, contributing to the knowledge of the area.
\end{abstract}

Keywords: Nursing. Philosophy. Research.

\section{Resumen}

Este artículo tuvo como objetivo evaluar las investigaciones, en el área de la Enfermería, que utilizaron la filosofía para comprender su práctica, identificando su contribución para la profesión. Es un estudio de revisión integrada, de carácter crítico y retrospectivo. Para seleccionar los artículos, utilizamos las bases de dados Lilacs y Medline. La muestra de esta revisión tuvo 31 artículos. Después de analizarlos, los resultados indicaron que los estudios cualitativos y reflexivos predominaron, utilizando la fenomenología como abordaje filosófica, predominando el referencial teórico del filósofo Martin Heidegger. Llegamos a la conclusión de que la fenomenología tiene sido cada vez más utilizada en trabajos realizados en el campo de la Enfermería, y esto refleja la preocupación en comprender los fenómenos vividos en su rutina. Así, se ha presentado importantes subvenciones para la práctica de la profesión y, especialmente, para edificar el conocimiento como disciplina.

Palabras clave: Enfermería. Filosofía. Investigación.

\footnotetext{
${ }^{1}$ Enfermeira. Mestre do Programa Associado de Pós-Graduação em Enfermagem da Universidade de Pernambuco (UPE) e Universidade Estadual da Paraíba (UEPB). Recife-PE. Brasil. E-mail: raquellcatunda@gmail.com; ²Enfermeira. Mestre do Programa Associado de Pós-Graduação em Enfermagem da Universidade de Pernambuco (UPE) e Universidade Estadual da Paraíba (UEPB). Campina Grande-PB. Brasil. E-mail: hemiliagoc@hotmail.com; ${ }^{3}$ Enfermeira. Mestre pelo Programa Associado de Pós-Graduação em Enfermagem da Universidade de Pernambuco (UPE) e Universidade Estadual da Paraíba (UEPB). Professora do Curso de Bacharelado em Enfermagem da Associação Caruaruense de Ensino Superior (ASCES). Recife-PE. Brasil. E-mail: samira.almeida@ig.com.br; ${ }^{4}$ Enfermeira. Doutora em Enfermagem pela Universidade de São Paulo/Escola de Enfermagem de Ribeirão Preto (USP/EERP). Coordenadora do Programa Associado de Pós-Graduação em Enfermagem da Universidade de Pernambuco (UPE) e Universidade Estadual da Paraíba (UEPB). Recife-PE. Brasil. E-mail: fatimabrao@terra.com.br; ${ }^{5}$ Enfermeiro. Editor Chefe da Escola Anna Nery Revista de Enfermagem. Professor Adjunto do Departamento de Enfermagem Fundamental. Docente do quadro permanente do Programa de Pós-Graduação da EEAN/UFRJ. Pesquisador do Núcleo de Pesquisa de História da Enfermagem Brasileira (NUPHEBRAS). Rio de Janeiro-RJ. Brasil. E-mail: ajafilhos@gmail.com; ${ }^{6}$ Socióloga e Economista. Doutora e Pós-Doutora em Educação Física/Sociologia das Emoções pela Universidade do Porto. Portugal. Docente da Universidade de Pernambuco (UPE). E-mail: clarasilvestre@uol.com.br
} 


\section{INTRODUÇÃO}

A enfermagem tem como essência o cuidado individual, familiar e comunitário com o ser humano, apresentando como principais metas: 0 desenvolvimento de intervenções de promoção da saúde, prevenção de doenças, recuperação e reabilitação da saúde, além de oferecer o conforto necessário, o acolhimento, bem-estar e autonomia dos pacientes no seu próprio cuidado".

Neste sentido, para conseguir realizar um cuidado integral, é de suma importância fazer uma reflexão acerca do contexto do cuidar diante do complexo processo de saúdedoença no qual o indivíduo está inserido, levando em consideração os muitos desafios que se apresentam neste cenário, a exemplo da tecnologia e da ciência que vêm, a cada dia, se desenvolvendo, com perspectiva de aumentar a sobrevida e a cura de doenças até então consideradas incuráveis. Porém, estes mesmos recursos passam a não considerar o indivíduo nos seus diversos aspectos, reduzindo-o ao cuidado fragmentado, centrado no tratamento da doença.

Para alcançar este objetivo, a Enfermagem sempre busca o saber visando renovar seus conceitos para beneficiar o indivíduo por meio de uma assistência que o contemple de forma holística. Contudo, compreender o ser humano é sempre uma tarefa complexa, pois envolve questões multidimensionais, fazendo-se relevante tomar posse dos recursos reflexivos propostos pela Filosofia, com vistas ao desenvolvimento de uma consciência crítica, participativa, além de uma prática profissional humanizada, a partir dos saberes apreendidos.

A palavra filosofia, criada por Pitágoras, é de origem grega e é formada pelas palavras: philo e sophia. A primeira significa amizade, amor fraterno; e a segunda significa sabedoria. Assim, a conexão destas palavras diz respeito à amizade pela sabedoria, amor e respeito pelo saber, sendo o filósofo todo aquele que ama a sabedoria, e tem anseio pelo saber².

Quem se dedica à filosofia, põe-se à procura do homem, escuta o que ele diz, observa o que ele faz e se interessa por sua palavra e ação, desejoso de partilhar, com seus concidadãos, do destino comum da humanidade. Eis porque a filosofia não se transforma em credo. Está em contínua pugna consigo mesma ${ }^{3: 138}$.

A filosofia consiste, portanto, em buscar a verdade, assim como em questionar e desvelar os fatos, analisandoos. É aprender a olhar o mundo e as pessoas com mais cuidado e responsabilidade, procurando compreender suas atitudes individual e coletivamente. Particularmente na Enfermagem, esta ciência colabora para uma atuação crítica do enfermeiro sobre toda a realidade que o circunda, visto que "uma filosofia para os enfermeiros guiará seu pensamento e ação", sendo incluídas as suas orientações sociais, espirituais e profissionais ${ }^{4: 175}$.

Diante da constante necessidade de reflexão crítica, é possível observar que a produção científica em Enfermagem no Brasil vem cada vez mais utilizando a filosofia como auxílio para tomada de decisão no seu cotidiano. Por isso, os pesquisadores vêm se motivando e assumindo o compromisso de publicar os resultados obtidos em seus estudos a fim de contribuir para o crescimento da Enfermagem.

Objetiva-se, portanto, neste estudo, realizar um levantamento das pesquisas, na área de Enfermagem, que fizeram uso da filosofia com perspectiva de compreender sua prática, buscando identificar sua contribuição para a profissão.

\section{PERCURSO METODOLÓGICO}

Trata-se de um estudo cuja base metodológica é a revisão integrativa, de caráter crítico e retrospectivo com fonte de dados secundários. Este tipo de estudo utiliza literaturas acerca de um tema predeterminado como fonte de dados, integrando as conclusões e achados das produções consultadas.

Inicialmente, a presente pesquisa foi projetada seguindo as seguintes etapas: definição do objeto de estudo; estabelecimento da estratégia de busca nas bases de dados; $\mathrm{e}$ seleção de estudos. Seguindo esta ordem, os artigos foram investigados nas Bases de Dados Lilacs e Medline, da Biblioteca Virtual em Saúde (BVS), adotando os descritores "Enfermagem" e "Filosofia", os quais foram cruzados com o auxílio do operador booleano AND, resultando na exibição de 6349 artigos. Destes, foram incluídos para análise as produções atuais em veículo on-line, em idioma português, disponíveis na íntegra, compreendendo o período de 1999 a 2009, com a finalidade de abranger as publicações dos últimos dez anos. Foram excluídos os artigos de revisão sistemática, os editoriais de periódicos, monografias, dissertações e teses por considerar a sua busca sistemática inviável logisticamente. As pesquisas repetidas foram contabilizadas apenas uma vez. Desta forma, chegou-se a um total de 94 publicações.

Em seguida, analisou-se o título e o resumo dos artigos, sendo selecionados aqueles que abordaram alguma temática relacionada à Filosofia nos artigos de Enfermagem. Realizouse, ainda,uma leitura individual de cada resumo, por dois revisores, verificando sua pertinência para serem ou não contabilizados neste estudo. Desta forma, chegou-se ao total de 31 publicações; destas, 24 provêm da Base de dados da Lilacs e 7 da Medline.

\section{APRESENTAÇÃO E DISCUSSÃO DE RESULTADOS}

Para organização dos resultados obtidos a partir da análise dos artigos no período de 1999 a 2009, foram elaborados dois quadros e dois gráficos. 0 Quadro 1 caracteriza os artigos encontrados pelo título da pesquisa e ano de publicação. 0 Quadro 2 contém as bases de dados 
e periódicos as quais pertencem. Os Gráficos 1 e 2 apresentam as abordagens filosóficas utilizadas e as áreas temáticas abordadas, respectivamente.

É possível notar que a enfermagem vem se desenvolvendo a cada ano e isso tem reflexo no aumento da produção científica na área, tanto em pesquisas quantitativas quanto nas que utilizam a abordagem qualitativa. Especificamente neste estudo, observa-se no Quadro 1 que, entre os artigos selecionados, o uso da filosofia em pesquisas na área de enfermagem tem sido gradativo, tendo sua produção aumentada a partir do ano de 2005, concentrando-se no ano de 2009.

Quadro 1: Caracterização dos artigos encontrados, 1999-2009.

\begin{tabular}{|c|c|c|}
\hline & TÍTULO & ANO \\
\hline Art. 1 & $\begin{array}{l}\text { Exxito témico, sucesso prático e sabedoria prática: bases conceituais } \\
\text { hermenêuticas para o cuidado de enfermagem à criança }\end{array}$ & 2009 \\
\hline Art. 2 & $\begin{array}{l}\text { Cuidado, autocuidado e cuidado de si: uma compreensão paradigmática para o } \\
\text { cuidado de enfermagem }\end{array}$ & 2009 \\
\hline Art. 3 & Pesquisa em enfermagem: possibilidades da filosofia de Buber & 2009 \\
\hline Art. 4 & $\begin{array}{l}0 \text { processo de elaboração de cartilhas para orientação do autocuidado no } \\
\text { programa educativo em Diabetes }\end{array}$ & 2009 \\
\hline Art. 5 & $\begin{array}{l}\text { Fenomenologia de Maurice Merleau-Ponty como referencial Teórico-filosófico } \\
\text { numa pesquisa de ensino em enfermagem }\end{array}$ & 2009 \\
\hline Art. 6 & $\begin{array}{l}\text { Reflexões sobre a filosofia do limite e suas implicações para o cuidar em } \\
\text { enfermagem }\end{array}$ & 2009 \\
\hline Art. 7 & $\begin{array}{l}\text { Cotidiano de mães-Acompanhantes-de-filhos-que-foram-a-óbito: contribuições para } \\
\text { a enfermagem oncológica }\end{array}$ & 2009 \\
\hline Art. 8 & $\begin{array}{l}\text { A visão da família sobre o trabalho de profissionais de saúde mental de um centro } \\
\text { de atenção psicossocial }\end{array}$ & 2009 \\
\hline Art. 9 & Fenomenologia & 2008 \\
\hline Art. 10 & Enfermagem como prática social: um exercício de reflexão & 2008 \\
\hline Art. 11 & 0 dito e o não-dito do ser-docente-enfermeiro/a na compreensão da sensibilidade & 2008 \\
\hline Art. 12 & Relações entre o familiar e criança com AIDS: compreensões à luz de Martin Buber & 2008 \\
\hline Art. 13 & Perspectiva problematizadora da educação popular em saúde e a enfermagem & 2007 \\
\hline Art.14 & $\begin{array}{l}\text { Processos de subjetivação inscritos na constituição da experiência de si da(o) } \\
\text { enfermeira(o) nas práticas assistenciais de um cenário de trabalho exemplar: a } \\
\text { Unidade de Terapia Intensiva }\end{array}$ & 2007 \\
\hline Art. 15 & A dimensão da subjetividade no processo de trabalho: visão arendtiana & 2007 \\
\hline Art. 16 & Processo clinical caritas: novos rumos para o cuidado de enfermagem transpessoal & 2006 \\
\hline Art. 17 & Na trilha da fenomenologia: um caminho para a pesquisa em Enfermagem & 2006 \\
\hline Art. 18 & A reforma psiquiátrica possível e situada & 2006 \\
\hline Art. 19 & A noção de cuidado de si mesmo e o conceito de autocuidado na enfermagem & 2006 \\
\hline Art. 20 & Fundamentos fenomenológicos para un cuidado comprensivo de enfermería & 2006 \\
\hline Art. 21 & 0 conhecimento científico como valor no agir do enfermeiro & 2005 \\
\hline Art. 22 & 0 cuidado de si como princípio ético do trabalho em enfermagem & 2005 \\
\hline Art. 23 & Que é o "ser da família"? & 2005 \\
\hline Art. 24 & $\begin{array}{l}\text { O cuidado na perspectiva de Leonardo Boff, uma personalidade a ser } \\
\text { (re)descoberta na enfermagem }\end{array}$ & 2005 \\
\hline Art. 25 & A dimensão do conceito em Deleuze e na enfermagem & 2004 \\
\hline Art. 26 & $\begin{array}{l}0 \text { cuidado de enfermagem na unidade de terapia intensiva (UTI): teoria } \\
\text { humanística de Paterson eZderad }\end{array}$ & 2004 \\
\hline Art. 27 & $\begin{array}{l}\text { Políticas públicas em relação à família, exigência institucional da filosofia política } \\
\text { de Hegel e de Marx }\end{array}$ & 2004 \\
\hline Art. 28 & Abordagem holística do termo pessoa em um estudo empírico: uma análise crítica & 2002 \\
\hline Art. 29 & $\begin{array}{l}\text { A formação da enfermeira na perspectiva da educação - reflexões e desafios para } \\
\text { o futuro }\end{array}$ & 2002 \\
\hline Art. 30 & A percepção do aluno sobre cuidado: reflexões dos pressupostos de Mayeroff & 2002 \\
\hline Art. 31 & $\begin{array}{l}\text { Evolução da disciplina de administração aplicada à enfermagem na escola de } \\
\text { enfermagem da USP no período de } 1980 \text { a } 1995\end{array}$ & 1999 \\
\hline
\end{tabular}


Esta estratégia de maior emprego da filosofia nos estudos corrobora para que a pesquisa em Enfermagem se configure como uma estratégia essencial para a geração de saberes, uma vez que subsidia a construção de um conhecimento cientificamente validado, permitindo uma intensa reflexão sobre a prática e fornecendo uma base de sustentação para o exercício do cuidar. Necessita, para isso, que o conhecimento produzido seja transmitido por meio da divulgação científica, através da qual a Enfermagem obtém ferramentas para se fortalecer enquanto ciência e melhorar a qualidade de vida humana ${ }^{5}$.
Na análise por periódicos, constatou-se que as revistas Texto \& Contexto Enfermagem e a Revista Brasileira de Enfermagem foram os periódicos que publicaram um número maior de artigos relacionados à temática abordada (Quadro 2). É possível que se deva ao fato de esta revista estar vinculada a um Programa de Pós-Graduação strictu sensu que apresenta grupos e linhas de pesquisa que promovem a construção de estudos embasados em reflexões críticas sobre a ciência e filosofia em relação aos conhecimentos e práticas produzidas na Enfermagem.

Quadro 2: Caracterização dos artigos encontrados, segundo a base e o periódico.

\begin{tabular}{|l|l|l|}
\hline & BASE & PERIÓDICO \\
\hline Art. 1 & LLACS & Revista Latino-Americana de Enfermagem \\
\hline Art. 2 & MEDLINE & Rev. esc. Enferm. - USP \\
\hline Art. 3 & LLACS & Revista Brasileira de Enfermagem \\
\hline Art. 4 & LLACS & Revista Brasileira de Enfermagem \\
\hline Art. 5 & LLLACS & Revista Gaúcha de Enfermagem \\
\hline Art. 6 & LLACS & Redalyc \\
\hline Art. 7 & LLACS & Esc. Anna Nery Rev. Enferm \\
\hline Art. 8 & LLACS & Esc. Anna Nery Rev. Enferm \\
\hline Art. 9 & MEDLINE & Revista Brasileira de Enfermagem \\
\hline Art. 10 & MEDLINE & Revista Brasileira de Enfermagem \\
\hline Art. 11 & MEDLINE & Revista Brasileira de Enfermagem \\
\hline Art. 12 & LLACS & Revista Gaúcha de Enfermagem \\
\hline Art. 13 & LLACS & Texto \& Contexto Enfermagem \\
\hline Art.14 & LLACS & Texto \& Contexto Enfermagem \\
\hline Art. 15 & LLACS & Ciência, Cuidado e Saúde \\
\hline Art. 16 & LLACS & Acta Paulista de Enfermagem \\
\hline Art. 17 & LLACS & Texto \& Contexto Enfermagem \\
\hline Art. 18 & LLACS & Rev. Enferm. UER \\
\hline Art. 19 & LLACS & Texto \& Contexto Enfermagem \\
\hline Art. 20 & LLACS & Texto \& Contexto Enfermagem \\
\hline Art. 21 & LLLACS & Rev. Esc. Enferm. - USP \\
\hline Art. 22 & LLACS & Texto \& Contexto Enfermagem \\
\hline Art. 23 & LLACS & Redalyc \\
\hline Art. 24 & MEDLINE & Revista Brasileira de Enfermagem \\
\hline Art. 25 & LLACS & Revista Latino-Americana de Enfermagem \\
\hline Art. 26 & MEDLINE & Revista Latino-Americana de Enfermagem \\
\hline Art. 27 & LLAACS & Online braz. J. nurs. (Online) \\
\hline Art. 28 & LLACS & Revista Latino-Americana de Enfermagem \\
\hline Art. 29 & LLACS & Rev. Esc. Enferm. USP \\
\hline Art. 30 & LLACS & Acta Paulista de Enfermagem \\
\hline Art. 31 & MEDLINE & Rev. Esc. Enferm. - USP \\
\hline & & \\
\hline
\end{tabular}

A partir de uma análise geral, viu-se também que há uma dominação de estudos qualitativos e reflexivos, predominantemente utilizando a fenomenologia como abordagem filosófica, quase que de forma exclusiva, como se observa no Gráfico 1. Isto prova que a Enfermagem vem intensificando os esforços no desenvolvimento das questões subjetivas do cuidado, contribuindo, desta forma, para o crescimento da profissão enquanto ciência e da melhoria da qualidade da assistência. Diante disso, optou-se por discutir a influência desta corrente filosófica na Enfermagem. 


\section{GRÁFICO 1. Caracterização dos artigos encontrados quanto à abordagem filosófica, 1999-2009.}

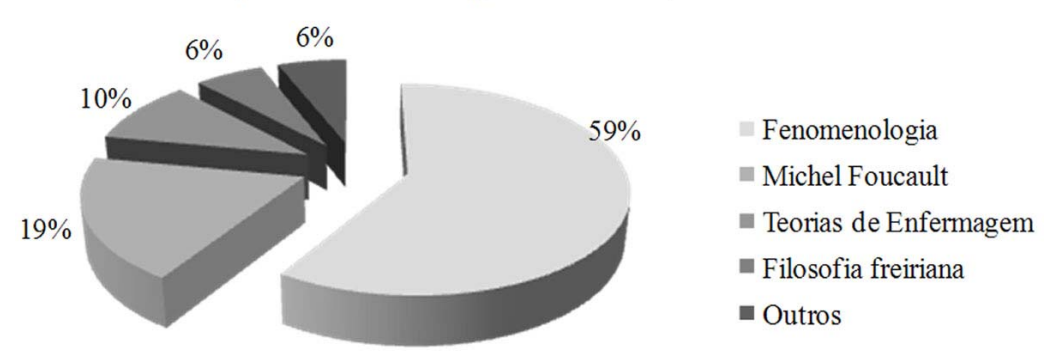

A história do desenvolvimento da pesquisa na Enfermagem Brasileira sofreu forte influência do positivismo, principalmente pelo modelo biomédico e abordagem quantitativa, porque, segundo esta corrente de pensamento, a única forma de conhecimento verdadeiro era o conhecimento científico ${ }^{6}$. Então, desconsideravam todas as outras formas do conhecimento humano que não podiam ser comprovadas ou experimentadas cientificamente. Como consequência, o agir/ cuidar da Enfermagem caracterizava-se pela valorização apenas dos aspectos técnicos, concretos e quantificáveis do cuidado.

Porém, apesar da sua forte influência, este modelo passou a concorrer com outros, devido a sua incapacidade de abranger os múltiplos fatores que interferem nas relações saúdedoença e no cuidar em Enfermagem. Fazia-se necessário, então, uma nova forma de ver o homem, de investigá-lo na sua totalidade, que contemplasse tanto os aspectos concretos como os aspectos subjetivos do cotidiano associado aos seus dilemas existenciais?.

Assim, por volta da década de 80, a Enfermagem inicia uma nova etapa, diferente daquela inspirada no modelo biomédico tradicional das ciências naturais e exatas calcado no método experimental, e se aproxima da pesquisa qualitativa que se volta para a compreensão dos sujeitos envolvidos no processo cuidar-cuidado ${ }^{5}$. Esta busca por novos horizontes de compreensão na Enfermagem refletiu em um movimento ao se voltar para influências filosóficas como a Fenomenologia, considerada mais adequada à compreensão da experiência humana ${ }^{8}$. Desta forma, acredita-se que a Enfermagem aproximou-se, conscientemente ou não, desta alternativa metodológica ${ }^{9}$, ocasionando o aumento do número de estudos de natureza qualitativa nos últimos anos.

A fenomenologia surge como uma filosofia interessada em estudar os procedimentos conscientes dependentes de objetivos universais, tais como aqueles existentes na matemática e na lógica. Inicia-se com a tentativa de descobrir um modo verdadeiramente filosófico de estudar a consciência que era redutível à psicologia ${ }^{10}$.

Este método é reconhecido enquanto ciência rigorosa desde 1900, mas no Brasil ainda tem sido pouco difundido já que suas raízes filosóficas nasceram na Europa a partir da crise das ciências. Entretanto, importantes iniciativas vêm sendo realizadas, sendo crescente o número de dissertações e teses que procuram viabilizar a sua práxis utilizando como norte de entendimento o método fenomenológico ${ }^{11}$.

0 objetivo deste tipo de pesquisa é descrever como fenômenos específicos de interesse são vivenciados e experimentados pelos indivíduos. 0 foco de seus estudos está no entendimento do que uma experiência representa no contexto de vida das pessoas ${ }^{12}$, voltando ao mundo-vida, na busca de um significado para as coisas revelado a partir das descrições desses sujeitos.

Muitos autores consideram que o método das ciências naturais não consegue responder "o que é mesmo a coisa" que está sendo pesquisada. Contudo, a fenomenologia não desvaloriza o método natural, mas explicita que ela examina processos a partir de um espectro causalista, estabelecendo leis nesses processos. ${ }^{13}$ Desta maneira, este tipo de abordagem propõe um método que explica o significado das coisas tal qual elas se manifestam, representado pela expressão máxima " volta as coisas nelas mesmas". Assim, este método "não caracteriza a quididade real dos objetos da investigação filosófica, o quê dos objetos, mas o seu modo, o como dos objetos"14:66.

Entre as vertentes desta metodologia, Martin Heidegger foi o filósofo que teve suas ideias mais utilizadas como referencial filosófico nos artigos selecionados. A fenomenologia heideggeriana possibilita um caminhar para o ser, pois pretende conhecer como o homem encontra-se vivendo alguma situação ou significando sua relação com o mundo. Desta forma, este tipo de pesquisa permite ao profissional de Enfermagem dar sentidos às suas vivências, contribuindo para que ele fique mais atento e reflexivo acerca da realidade e o modo de ser do outro ${ }^{15}$.

Então, ao compreender que a abordagem fenomenológica estuda a descrição dos fenômenos do ser humano e seus significados, a produção da Enfermagem nesta área se revela no caminho da crítica, da reflexão e da busca pelos significados no intuito de compreender 0 seu fazer ${ }^{8}$. Assim, esta passou a despertar a atenção de pesquisadores de Enfermagem como uma alternativa de método de investigação, e, embora não tenham sido 
elaborados aspectos específicos para a Enfermagem pelos grandes filósofos, eles abordaram pontos de interesse para a profissão, contemplando as inquietações de quem centra o foco do seu cuidar no ser humano ${ }^{10,16}$.
Várias são as áreas abordadas nas pesquisas queutilizaram a fenomenologia como abordagem filosófica, entre elas: saúde da criança, saúde mental, saúde do trabalhador, saúde da família, saúde pública e educação em Enfermagem (Gráfico 2).

\section{GRÁFICO 2. Caracterização dos artigos encontrados} quanto à área temática, 1999-2009.
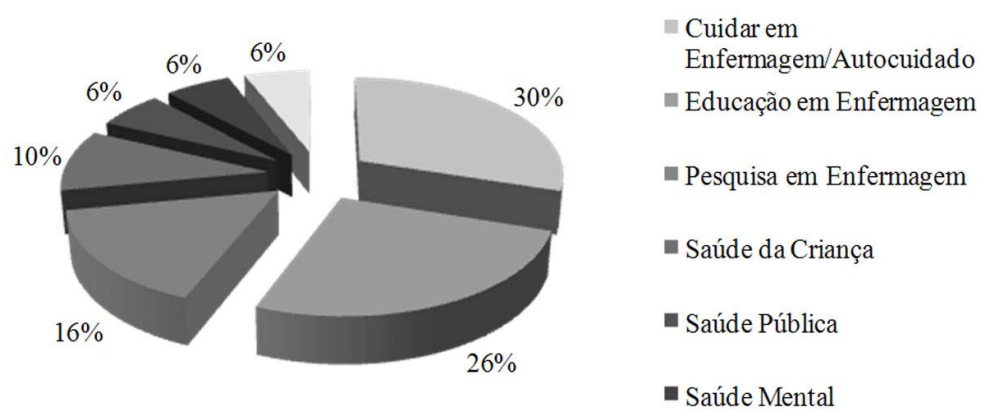

Esta diversidade de áreas temáticas sugere que a preocupação com o indivíduo não se restringe a um único setor de atuação da profissão, contemplando aquele que é cuidado de forma global.

Neste ínterim, ao passo que a Enfermagem procurou compreender o ser em sua dimensão holística, abrindo o campo de possibilidades do cuidado de forma integral. Então, ao lidar com questões existenciais dos indivíduos cuidados, apropriou-se da fenomenologia como base para suas reflexões e práticas em um mergulho na subjetividade e na essência que envolve o cuidar $^{16}$.

É possível que essa subjetividade seja alcançada no contexto que envolve a prática diária:

A enfermagem, em seu cotidiano, interage continuamente com o homem doente, seja através de gestos, palavras ou até mesmo de um simples olhar, realizando um verdadeiro partilhar. Nesse ENCONTRO entre pessoas, o DIÁLOGO pode ser considerado um momento especial onde se exteriorizam as expectativas, os anseios e temores; enfim, a perspectiva que o cliente tem do mundo e de sua situação atual (SER-DOENTE) $)^{17: 407}$.

Entende-se que as ações no contexto da Enfermagem são desenvolvidas com os sujeitos que estão inseridos no mundo social, o qual está em constante movimento por meio das relações sociais e das necessidades específicas de cada ser envolvido nessas relações. Desta forma, é possível dizer que a Enfermagem estabelece uma relação intencional entre indivíduos no mundo da vida e profissional. Portanto, esta prática que leva em conta a subjetividade e a relação social permite a compreensão das necessidades do indivíduo e o estabelecimento de uma relação de confiança para com o enfermeiro. Assim, deve-se valorizar a escuta e consentir que o outro fale seus anseios e angústias, além da necessidade de aceitar as diferenças e não ter conceitos pré-formulados em seu cotidiano ${ }^{18}$.

Portanto, por lidar com questões existenciais do ser humano, a enfermagem tem na fenomenologia uma enorme contribuição para o seu pensar e fazer, uma vez que, para alcançar a compreensão dos acontecimentos do cotidiano, é necessário que saiba imergir na subjetividade e sua essência sem, contudo, esquecer da objetividade que o envolve ${ }^{5}$.

\section{CONSIDERAÇÕES FINAIS}

Acerca da produção científica de Enfermagem, no Brasil, observa-se que, cada vez mais, os pesquisadores vêm se motivando e assumindo o compromisso de publicar os resultados obtidos em seus estudos, inclusive os que inserem a Filosofia em seu contexto.

A fenomenologia tem sido uma abordagem filosófica cada vez mais utilizada em trabalhos realizados no campo da Enfermagem, o que reflete a preocupação em compreender os fenômenos vividos no seu dia-a-dia, entendendo a complexidade dos mesmos, bem como a singularidade expressa por cada sujeito participante. Neste sentido, tem apresentado subsídios expressivos para a práxis da profissão e, especialmente, para a edificação do conhecimento enquanto disciplina.

Assim, ao buscar compreender o significado da experiência vivida do homem, tem fornecido elementos impor tantes para o conhecimento das várias dimensões que permeiam o cuidado no processo de viver humano.

Desta forma, esta ciência tem um papel muito importante nestes estudos, visto que proporciona questionamentos, buscando investigá-los e dar respostas, 0 que para a Enfermagem se faz necessário, para que sejam realizadas reflexões acerca da prática cotidiana e, consequentemente, aprimorar nossas ações, beneficiando, em 
primeiro lugar, o atendimento de qualidade aos usuários dos serviços de saúde.

\section{REFERÊNCIAS}

1.Barbosa MA, Medeiros M, Prado MA, Bachion MM, Brasil W. Reflexões sobre o trabalho do enfermeiro em saúde coletiva. Rev Eletr Enferm. 2004;6(1): 9-15.

2.Chaui M. Convite à filosofia. $12^{\mathrm{a}}$ ed. São Paulo: Ática; 2000.

3.Jaspers Karl. Introdução ao pensamento filosófico. São Paulo: Pensamento/Cultrix; 1965.

4.Ferlic A. Abordagem existencialista na enfermagem. Enferm Nov Dimens. 1976;2(3): 175-81.

5.Almeida IS, Crivaro ET, Salimena AMO, Souza IEO. 0 caminhar da enfermagem em fenomenologia: revisitando a produção acadêmica. Rev Eletr Enferm. [on-line]. 2009; [citado 2010 ago]; 11(3): 695-99. Disponível em: http://www.fen.ufg.br/revista/v11/n3/pdf/v11n3a30.pdf.

6.Lino MM, Backes VMS, Ferraz F, Reibnitz KS, Martini JG . Análise da produção cientíica dos grupos de pesquisa em educação em enfermagem na região sul do Brasil. Texto\&Contexto Enferm. 2010 abr/jun; 19(2): 265-73.

7.Gil AC, Licht RHG, Santos BRMS. Por que fazer pesquisa qualitativa em saúde? Caderno de Pesquisa em Ciências da Saúde. 2006;1 (2): 5-19.

8. Carvalho MDB, Valle ERM. A pesquisa fenomenológica e a enfermagem. Acta Sci; Health Sci. 2002; 24(3): 843-47.

9.Capalbo C. Considerações sobre o método fenomenológico e a enfermagem. Rev Enferm UERJ. 1994 out; 2 (2):192-97.

10.Martins J, Bicudo MAV. A pesquisa qualitativa em psicologia. $5^{\mathrm{a}}$ ed. São Paulo: Centauro; 2005.

11.Pokladek DD. A fenomenologia do cuidar: prática dos horizontes vividos nas áreas da saúde, educacional, e organizacional. São Paulo: Vetor; 2004.

12.Driessnack M, Souza VD, Mendes IAC. Revisão dos desenhos de pesquisa relevantes para enfermagem: Parte 2: desenhos de pesquisa qualitativa. Rev Latino-Am Enfermagem. 2007 jul/ago; [ citado ago]: 15(4). Disponível em: <http://www.scielo.br/scielo.

13.Josgrilberg R. de. A fenomenologia como novo paradigma de uma ciência do existir. In: Pokladek DD. A fenomenologia do cuidar: prática dos horizontes vividos nas áreas da saúde, educacional e organizacional. São Paulo: Vetor; 2004. p. 31-52.

14. Heidegger M. Ser e tempo. Tradução de Márcia de Sá Cavalcante. $4^{\text {a }}$ ed. Petrópolis(RJ): Vozes; 2009.

15.Monteiro CFS, Rocha SS, Paz EPAl, Souza EO. Fenomenologia heideggeriana e sua possibilidade na construção de estudos de enfermagem. Esc Anna Nery. 2006 ago; 10(2): 297-300.
16. Terra $M G$, et al. Na trilha da fenomenologia: um caminho para a pesquisa em enfermagem. Texto\& Contexto Enferm. 2006 out-dez; 15(4): $672-78$.

17.Spindola T. A fenomenologia e a enfermagem: algumas reflexões. Rev Esc Enferm USP. 1997 dez; 31 (3):403-09.

18.Tocantins FR; Nogueira ML. Abordagem fenomenológica de Alfred Schutz na enfermagem. In: Pokladek DD. A fenomenologia do cuidar: prática dos horizontes vividos nas áreas da saúde, educacional e organizacional. São Paulo: Vetor; 2004. p. 61-68. 